\title{
ON DISTRIBUTIVELY GENERATED NEAR-RINGS ${ }^{1}$
}

\author{
by STEVE LIGH \\ (Received 16th May 1968)
}

The following theorems in ring theory are well-known: identity.

1. Let $R$ be a ring. If $e$ is a unique left identity, then $e$ is also a right

2. If $R$ is a ring with more than one element such that $a R=R$ for every nonzero element $a \in R$, then $R$ is a division ring.

3. A ring $R$ with identity $e \neq 0$ is a division ring if and only if it has no proper right ideals.

In this note we shall show that the above theorems can be generalized to distributively generated near-rings. Examples will be given to show that the theorems do not hold for arbitrary near-rings.

\section{Definitions}

A near-ring $R$ is a system with two binary operations, addition and multiplication, such that:

(i) The elements of $R$ form a group $R^{+}$under addition.

(ii) The elements of $R$ form a multiplicative semi-group.

(iii) $x(y+z)=x y+x z$, for all $x, y, z \in R$.

In particular, if $R$ contains a multiplicative semigroup $S$ whose elements generate $R^{+}$and satisfy

(iv) $(x+y) s=x s+y s$, for all $x, y \in R$ and $s \in S$,

we say that $R$ is a distributively generated (d.g.) near-ring.

The most natural example of a near-ring is given by the set $R$ of all mappings of an additive group (not necessarily abelian) into itself. If the mappings are added by adding images and multiplication is iteration, then the system $(R,+,$. is a near-ring. If $S$ is a multiplicative semigroup of endomorphisms of $G$ and $R^{\prime}$ is the sub-near-ring generated by $S$, then $R^{\prime}$ is a d.g. near-ring. Other examples of d.g. near-rings may be found in (1).

A near-ring $R$ that contains more than one element is said to be a division near-ring if and only if the set $R^{\prime}$ of nonzero elements is a multiplicative group. Every division ring is an example of a division near-ring. For examples of division near-rings which are not division rings, see (4).

1 Portions of this paper appear in the author's Ph.D. dissertation written under the direction of Professor J. J. Malone, Jr., at Texas A\&M University. 
An element $a$ of $R$ is right distributive if $(b+c) a=b a+c a$ for all $b, c \in R$. An element $x \in R$ is anti-right distributive if $(y+z) x=z x+y x$ for all $y, z \in R$. It follows at once that an element $a$ is right distributive if and only if $(-a)$ is anti-right distributive. In particular, any element of a d.g. near-ring is a finite sum of right and anti-right distributive elements.

A subset $B$ of a near-ring $R$ is called a right ideal if $(B,+)$ is a subgroup of $(R,+)$ and $B . R=\{b . r: b \in B, r \in R\} \subseteq B$.

(1.1) Lemma. Let $R$ be a near-ring, then

(i) $x \cdot 0=0, x \in R$,

(ii) $x(-y)=-(x y), x, y \in R$.

In particular, if 1 is the identity of $R$, then

(iii) $x(-1)=-x, x \in R$.

These results are easy consequences of the definitions.

\section{Division near-rings}

In general if a near-ring has an identity $1,(-1)$ need not commute with all the elements. The following lemma is easy to verify:

(2.1) Lemma. If $R$ is a near-ring with identity 1 , then $(-1)(-1)=1$. Furthermore if $(-1) r=r(-1)$ for all $r \in R$, then $R^{+}$is commutative.

(2.2) Theorem. The additive group $R^{+}$of a division near-ring $R$ is abelian.

Proof. Observe that if $1+1=0$, then $x+x=x(1+1)=x .0=0$ for each non-zero element $x \in R$ and hence $R^{+}$is clearly abelian. If $(-1) \neq 1$, let $F$ be the mapping of $R$ into $R$ given by $r F=r(-1)+(-1) r . \quad F$ is a one-toone map. Suppose $r(-1)+(-1) r=s(-1)+(-1) s$. Then

$$
s+r(-1)+(-1) r+(-1) s(-1)=0 .
$$

It follows that $(-1)(r+s(-1))=r+s(-1)$. If $r+s(-1) \neq 0$, then $(-1)=1$, contrary to assumption. Thus $r+s(-1)=0$ and this implies $r=s$. Now if $R$ is finite, then $F$ is also an onto mapping which means that for $r \in R$, there is an element $s \in R$ such that $s(-1)+(-1) s=r$ or $r(-1)=(-1) s(-1)+s$. Hence $(-1)[s(-1)+(-1) s]=(-1) r$ implies $(-1) s(-1)+s=(-1) r$ and for all $r \in R$ we have $(-1) r=r(-1)$. From $(2.1), R^{+}$is abelian. This result was first proved by Zassenhaus (4). A proof for the infinite case can be found in (3).

Even if the additive group of a near-ring with identity 1 is commutative, $(-1)$ need not commute multiplicatively with all elements. For example, if $G$ is the additive abelian group of order three then the set of mappings defined on $G$ is a near-ring whose additive group is abelian. But $(-1) f \neq f(-1)$ where $f$ is a non-zero constant mapping. However this is true for "most" division near-rings as the following corollary shows:

(2.3) Corollary. Let $R$ be a division near-ring with identity 1 such that 1. $r=r .1$ for all $r \in R$, then $(-1) r=r(-1)$. 
Proof. Suppose there exists $w \in R$ such that $(-1) w=w(-1)+x, x \neq 0$. Then $x=w+(-1) w=(-1)((-1) w+w)=(-1)(w+(-1) w)=(-1) x$ and hence $(-1)=1$. Thus $w=w+x$ and this implies $x=0$, which is a contradiction.

Remark. It can be shown that if a division near-ring $R$ has three or more elements, then the identity on the multiplicative group is the identity on $R$.

\section{Distributively generated near-rings}

(3.1) Lemma. Let $R$ be a near-ring. If $u x=x$ for all $x \in R$, and if $a$ is anti-right distributive, then

(i) $(x+y+z) a=z a+y a+x a$,

(ii) $(x u+y+u) a=a$ where $x+y=y+x=0$.

Proof. Obvious.

(3.2) Theorem. If $R$ is a d.g. near-ring and if $u$ is a unique left identity, then $u$ is also a right identity.

Proof. Suppose $u x=x$ for all $x \in R$. Since $R$ is a d.g. near-ring, we have for any $w \in R, w=w_{1}+w_{2}+\ldots+w_{n}$ where $w_{i}$ is either a right or anti-right distributive element of $R$. Now consider $(x u+y+u) w$ where $x+y=y+x=0$ and $w$ is any element of $R$. Now applying (3.1) we have

$$
\begin{aligned}
(x u+y+u) w & =(x u+y+u)\left(w_{1}+w_{2}+\ldots+w_{n}\right) \\
& =(x u+y+u) w_{1}+(x u+y+u) w_{2}+\ldots+(x u+y+u) w_{n} \\
& =w_{1}+w_{2}+\ldots+w_{n} \\
& =w .
\end{aligned}
$$

The uniqueness of $u$ implies $x u=x$ for all $x \in R$. This completes the proof.

Remark. It can be shown easily that if a near-ring has a unique right identity, then it is also a left identity. Theorem (3.2) is not true in general for arbitrary near-rings. Consider the following example: Let $G$ be an additive group with at least three elements. Suppose $e \in G$ such that $e \neq 0$. Define $e x=x$ for all $x \in G$ and $g x=0$ for all $g \neq e$ of $G$. Then $(G,+,$. is a near-ring (2). It is clear that $e$ is the unique left identity but not a right identity.

The following lemma is easy:

(3.3) Lemma. If $D$ is a d.g. near-ring, then 0 . $d=0$ for all $d \in D$.

(3.4) Theorem. A necessary and sufficient condition for a d.g. near-ring $D$ with more than one element to be a division ring is that, for all nonzero $a \in D$, $a D=D$.

Proof. Necessity. There is an element $e \in D$ such that $a e=e a=a$ for $a \neq 0$ in $D$. Clearly $a D \subseteq D$. Suppose $a \neq 0$ is in $D$. Then there exists an 
element $b \in D$ such that $a b=e \in a D$. Thus $x=a(b x)$, for all $x \in D$, and so $x \in a D$. Hence $a D=D$.

Sufficiency. If $a$ and $b$ are nonzero elements of $D$, then $a b \neq 0$. For if not, there exist $a_{e}$ and $b_{e}$ such that $a a_{e}=a$ and $b b_{e}=a_{e}$. Thus

$$
0=a b b_{e}=a a_{e}=a \text {, }
$$

which is a contradiction. Now let $r$ be a nonzero right distributive element of $D$. Then there is an element $e \in D$ such that $r e=r$. But

$$
r(e r-r)=r e r-r r=0 .
$$

From the above we have $e r=r$. This means that $e$ is a two-sided identity for $r$. Since we know from the first part of the proof that the set of non-zero elements is closed under multiplication and multiplication is associative it only remains to prove that $e$ is a right identity for the non-zero elements of $D$ and every non-zero element of $D$ has a right inverse. Let $d \neq 0$ be an element in $D$. Then $(d e-d) r=d e r-d r=d r-d r=0$. Since $r \neq 0$, we have that $d e=d$. Also $d D=D$ implies there is a $d^{\prime} \in D$ such that $d d^{\prime}=e$. Thus we have shown that the d.g. near-ring $D$ is a division near-ring. From (2.2) the additive group $D^{+}$of $D$ is abelian. It now follows $(1$, p. 93) that every element of $D$ is right distributive and hence $D$ is a division ring.

(3.5) Corollary. A d.g. near-ring $D$ with identity $e \neq 0$ is a division ring if and only if it has no proper right ideals.

Proof. Necessity is quite clear. Suppose $D$ has no proper right ideals. For each $a \neq 0$ in $D, a D$ is a right ideal of $D$. Thus $a D=D$ and by (3.4) $D$ is a division ring.

The following example shows that (3.4) can not be extended to arbitrary near-rings: Let $D=\{0,1\}$ with addition and multiplication as defined below. Then it can be verified easily that $D$ is a near-ring which is not a division ring.

\begin{tabular}{l|ll}
+ & 0 & 1 \\
\hline 0 & 0 & 1 \\
1 & 1 & 0
\end{tabular}

\begin{tabular}{l|ll}
$\cdot$ & 0 & 1 \\
\hline 0 & 0 & 1 \\
1 & 0 & 1
\end{tabular}

In fact, $D$ is the only (up to isomorphism) division near-ring for which 1 is not the identity of $D$.

Finally it can be shown easily that a near-ring $D$ with identity $e \neq 0$ and $0 . x=0$ for all $x \in D$ is a division near-ring if and only if it has no proper right ideals. Since there exist division near-rings which are not division rings (4), we conclude that (3.5) can not be extended to arbitrary near-rings.

The author is grateful to the referee for his helpful suggestions.

\section{REFERENCES}

(1) A. Frohlich, Distributively generated near-rings (I. Ideal Theory), Proc. London Math. Soc. (3) 8 (1958), 76-94. 


\section{ON DISTRIBUTIVELY GENERATED NEAR-RINGS}

(2) J. J. Malone, Jr., Near-rings with trivial multiplications, Amer. Math. Monthly, 74 (1967), 1111-1112.

(3) B. H. Neumann, On the commutativity of addition, J. London Math. Soc. 15 (1940), 203-208.

(4) H. Zassenhaus, Über endlich Fastkorper, Abh. Math. Sem. Univ. Hamburg, 11 (1936), 187-220.

Department of Mathematics

TeXas A \& M UNIVERSITY

College Station, Texas 77843

U.S.A. 